\title{
In vivo and in situ imaging of head and neck squamous cell carcinoma using near-infrared fluorescent quantum dot probes conjugated with epidermal growth factor receptor monoclonal antibodies in mice
}

\author{
KAI YANG ${ }^{1}$, CHENG ZHAO $^{1}$, YU-AN CAO $^{2}$, HONG TANG $^{1}$, YUN-LONG BAI $^{1}$, \\ HAO HUANG ${ }^{1}$, CHUEN-RONG ZHAO ${ }^{1}$, RUI CHEN ${ }^{1}$ and DAN ZHAO ${ }^{1}$ \\ ${ }^{1}$ Department of Oral and Maxillofacial Surgery, The First Affiliated Hospital, Chongqing Medical University, Chongqing \\ 400016; ${ }^{2}$ Department of Stomatology, Second Affiliated Hospital of Soochow University, Suzhou 215004, P.R. China
}

Received December 19, 2011; Accepted January 20, 2012

DOI: $10.3892 /$ or.2012.1705

\begin{abstract}
In this study, we applied near-infrared fluorescent quantum dots (NIRF-QDs) for non-invasive in vivo and in situ imaging of head and neck squamous cell carcinoma (HNSCC). The U14 squamous cancer cell line with high expression of epidermal growth factor receptor (EGFR) was implanted subcutaneously into the head and neck regions of nude mice to establish HNSCC models. NIRF-QDs with an emission wavelength of $800 \mathrm{~nm}$ (NIRF-QD800) were conjugated with EGFR monoclonal antibodies to develop the QD800-EGFR $\mathrm{Ab}$ probe. In vivo and in vitro studies demonstrated that the QD800-EGFR Ab probe can specifically bind EGFR expressed on U14 cells. U14 squamous cell carcinoma in the head and neck can be clearly visualized by in vivo imaging after intravenous injection of QD800-EGFR Ab probes. The results suggested that in situ imaging using NIRF-QD-EGFR $\mathrm{Ab}$ probes has unique advantages and prospects for the investigation of tumor development, early diagnosis and personalized therapy of HNSCC.
\end{abstract}

\section{Introduction}

Non-invasive in vivo and in situ imaging of tumor cells plays an important role in studying the occurrence and development of cancers, making early diagnosis and conducting personalized therapies. This has been a difficult area due to the lack of highly sensitive imaging substances and specific marker for each particular type of tumor. Quantum dots (QDs) were

Correspondence to: Dr Kai Yang, Department of Oral and Maxillofacial Surgery, The First Affiliated Hospital, Chongqing Medical University, Chongqing 400016, P.R. China

E-mail: cqfyyk@hotmail.com

Key words: head and neck cancer, quantum dot, in vivo imaging developed recently and have shown great prospect in the noninvasive imaging of tumors $(1,2)$.

QD is a type of nanocrystal composed of elements belonging to II-VI or III-V groups with a diameter of 2-10 nm. In comparison to the conventional organic fluorescent dyes and fluorescent proteins, QDs have unique optical properties, e.g., broad and continuous distribution of the excitation spectrum, narrow and symmetric emission spectrum, strong fluorescence and high photochemical stability. In addition, QDs are less prone to photobleaching and the spectrum of any points from ultraviolet to the near infrared can be obtained by changing the size and composition of QDs $(3,4)$. These optical properties are not owned by all the current fluorescent probes. Currently, QDs have been applied in the imaging of biological macromolecules and cells both in vitro and in vivo (5-7). Non-invasive in vivo imaging has been used for the investigation of tumor development (8-10), early diagnosis of cancer (10), the transportation of drugs in vivo (11), and monitoring of therapeutic responses in vivo (11-13). These studies have demonstrated the unique advantages of QDs in the imaging of cells both in vitro and in vivo. Particularly, the recently developed near-infrared fluorescent quantum dot (NIRF-QD) with an emission range of 700-900 $\mathrm{nm}$ has the advantage of avoiding the interference of tissue auto fluorescence (400-600 nm). NIRF-QDs have strong tissue penetration, but do not have radiation and are not harmful in vivo. Therefore, NIRF-QDs are extremely suitable for non-invasive in vivo imaging $(14,15)$.

NIRF-QDs were conjugated with prostate specific membrane antigen monoclonal antibody (PSMA), Her2 monoclonal antibody and EGF monoclonal antibody, and have been used for non-invasive in vivo imaging of prostate cancer, breast cancer and colon cancer, respectively, in mice (16-18). The results from these studies have shown that NIRF-QD probes conjugated with monoclonal antibodies can specifically bind the corresponding antigens expressed by the tumor cells and in vivo imaging can be subsequently obtained. Our previous studies (10) have also shown that in vivo imaging can detect a minimum of $10^{4}$ NIRF-QDs-labeled cancer cells in the presence of skin barrier, which was 100-fold higher than 
the minimal detection limit of CT and MRI. These studies demonstrate that NIRF-QDs have unique advantages for early diagnosis and personalized therapy (e.g., determination of surgical margins, evaluation of the efficacy of targeted therapy, etc.). Targeting of the nano-probes to the tumors after intravenous injection is closely related to the location of the tumors. Currently, there are no studies on non-invasive in vivo and in situ imaging of head and neck tumors by using NIRF-QD conjugated with monoclonal antibodies.

The U14 squamous cancer cells in Kunming mice highly express EGFR $(19,20)$. In this study, we first developed head and neck squamous cell carcinoma model by implanting U14 cells subcutaneously into the chin-neck region of nude mice. We then conjugated EGFR monoclonal antibody to NIRF-QDs with a maximum emission wavelength of $800 \mathrm{~nm}$ to produce QD800-EGFR Ab probes. In situ and in vivo imaging of U14 squamous cell carcinoma can be obtained by intravenous injection of QD800-EGFR Ab probes. To our knowledge, this is the first study that EGFR antibody-conjugated NIRF-QDs were utilized for non-invasive in vivo and in situ imaging of head and neck squamous cell carcinoma (HNSCC). The results obtained from these studies provide foundations for the application of NIRF-QDs in vivo imaging, personalized diagnosis and treatment of HNSCC.

\section{Materials and methods}

Cell line and animals. The U14 cell line was purchased from the Chinese Academy of Medical Sciences Cancer Institute. The cells were maintained at $37^{\circ} \mathrm{C}$ in a humidified atmosphere containing $5 \% \mathrm{CO}_{2}$ in DMEM supplemented with $10 \%$ fetal bovine serum, $2 \mathrm{mM}$ L-glutamine, $100 \mathrm{U} / \mathrm{ml}$ penicillin, and $100 \mathrm{mg} / \mathrm{ml}$ of streptomycin. SPF level BALB/c nu/nu nude mice ( $n=15$, age, 6-8 weeks; weigh, 20-25 g) were purchased from the Experimental Animal Center of Chongqing Medical University and were maintained in constant temperature and humidity. Feeds, bedding and water were sterilized. All experimental procedures were approved by the Animal Administration Committee of Chongqing Medical University.

Preparation and purification of QD800-EGFR Ab probes. QD800-EGFR Ab probes were prepared using Qdot $^{\circledR}$ Antibody Conjugation Kits (Invitrogen, Carlsbad, CA, USA) according to the manufacturer's instruction. The first step was the activation and elution of QDs. In this step, $14 \mu 1$ SMCC at the concentration of $10 \mathrm{mM}$ was mixed with $125 \mu \mathrm{l}$ QD800 (amine-functionalized CdSeTe/ZnS) at the concentration of $4 \mu \mathrm{M}$. After activation at room temperature for $1 \mathrm{~h}$, the mixture was loaded onto NAP-5 column and the colored elution $(500 \mu \mathrm{l})$ was collected. The second step was antibody reduction and separation. In this step, 6.1 $\mu$ l DTT $(1 \mathrm{M})$ was added into $300 \mu \mathrm{l}$ EGFR monoclonal antibody (Abcam, UK) ( $1 \mathrm{mg} / \mathrm{ml}$ in PBS). After 30 -min reduction reaction, dye indicator was added and colored solution $(500 \mu \mathrm{l})$ was obtained by eluting from NAP-5 column. The third step was conjugation and inactivation. In this step, elutions collected in step 1 and 2 were mixed, and after 1-h conjugation, $3 \mu 1$ 2-mercaptoethanol (10 $\mathrm{mM})$ was added for 30-min inactivation. The final step was concentration and purification. In this step, the inactivated solutions were added into ultrafiltration tube. After $15 \mathrm{~min}$ of ultracentrifugation at 7,000 rpm, the conjugated solution was collected from the inner side of the ultrafiltration membrane. The conjugated solution was separated by chromatography and the purified QD800-EGFR Ab probes were obtained. The concentration of QD800-EGFR Ab probes was calculated by the equation: $\mathrm{A}=\varepsilon \mathrm{cl}$, where $\mathrm{A}$ represent absorbance, $\varepsilon$ represent the extinction coefficient, $\mathrm{c}$ represent the molar concentration and 1 represent the optical path.

The extinction coefficient at $550 \mathrm{~nm}\left(\varepsilon_{550}\right)$ of the purified QD800-EGFR Ab probes was $1.7 \times 10^{6}(\mathrm{~mol} / 1)^{-1} \mathrm{~cm}^{-1}$. Absorbance was measured by UV spectrophotometer (Beckman, DU-600) at $550 \mathrm{~nm}$ and the value was 3.442 and the optical path (1) was $1 \mathrm{~cm}$. According to the equation described in Materials and methods, the concentration of the purified QD800-EGFR Ab probes was $2.025 \mu \mathrm{M}$.

In vitro labeling of U14 cells by QD800-EGFR Ab probes. Well-grown U14 cells were inoculated into $935-\mathrm{mm}$ glass bottom culture dishes $(\varnothing 15 \mathrm{~mm})$ at a concentration of $5 \times 10^{4} /$ dish. After $24 \mathrm{~h}$ of culture, the medium was discarded, and the cells were washed with PBS 3 times. The cells were divided into 3 groups. In the experimental group, the cells were added with $100 \mu \mathrm{l}$ QD800-EGFR Ab probes (100 $\mathrm{nM})$. The cells in the control group I were added with $100 \mu$ l QD800 (100 nM). The cells in the control group II were added with $200 \mu \mathrm{l}$ EGFR monoclonal antibody $(1 \mu \mathrm{g} / \mathrm{ml})$ to block EGFR. After $2 \mathrm{~h}$ of incubation, the cells were washed with PBS 3 times and then added with $100 \mu \mathrm{l}$ QD800-EGFR Ab probes (100 nM). The cells in each group after addition of QD800-EGFR Ab probes or QD800 were incubated at $37^{\circ} \mathrm{C}$ for $30 \mathrm{~min}$ followed by 3 washes with PBS. The QD800-EGFR Ab probe-labeled U14 cells were observed under a confocal laser scanning microscope (Leica, TCS-SP5). The following scanning parameters were used: excitation, $405 \mathrm{~nm}$; emission, 750-800 nm; image acquisition size, $1024 \times 1024$ pixels; speed, $400 \mathrm{HZ}$; pinhole size, $135.9 \mu \mathrm{M}$; line average, 4; frame average, 1; zoom, 2.0; Scan-Direction, 2; resolution, 8 bits.

Development of HNSCC model in nude mice. Logarithmic growth phase U14 cells were trypsinized by $0.5 \%$ trypsin. After centrifugation at $4{ }^{\circ} \mathrm{C}(800 \mathrm{rpm})$ for $4 \mathrm{~min}$, the cells were resuspended in PBS. The suspension containing $2 \times 10^{6} \mathrm{U} 14$ cells were injected subcutaneously into the chin-neck junction area of 15 nude mice to establish the HNSCC model. Tumor growth was observed daily and the experiment started when the maximum diameter of the tumor reached $0.8-1.2 \mathrm{~cm}$.

In vivo imaging of the tumors. The mice with tumors were divided into experimental group, control group I and control group II with 5 mice in each group. After anesthesia by intraperitoneal injection of $2 \%$ sodium pentobarbital $(40 \mathrm{mg} / \mathrm{kg})$, each mouse in the experimental group was injected with $100 \mu \mathrm{l}$ QD800-EGFR Ab probes containing 100 pmol equivalent of QD800 via the tail vein. For the control group I, each mouse was injected with $100 \mu \mathrm{l}$ of QD800 containing 100 pmol equivalent of QD800 via the tail vein. Each mouse in the control group II was injected with $250 \mu$ l EGFR monoclonal antibody $(1 \mathrm{mg} / \mathrm{ml})$ to block EGFR. After $24 \mathrm{~h}$, the mice were injected with $100 \mu \mathrm{l}$ QD800-EGFR Ab probe containing 100 pmol equivalent of QD800 via the tail vein. In vivo imaging was 
Bright-field

$\mathbf{A}$

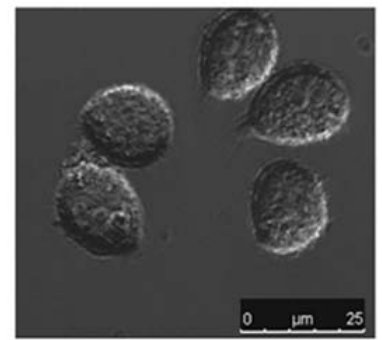

B

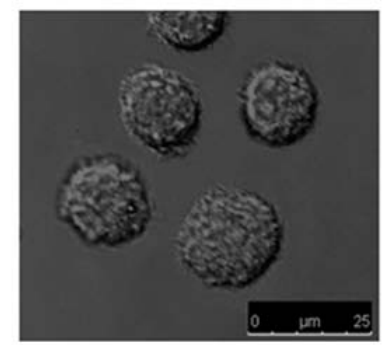

C

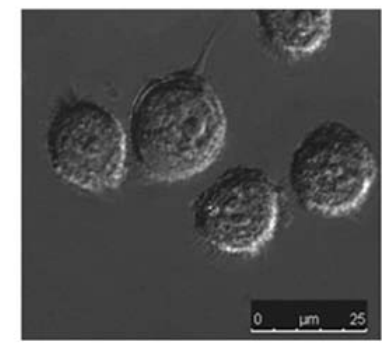

QD800 fluorescence image
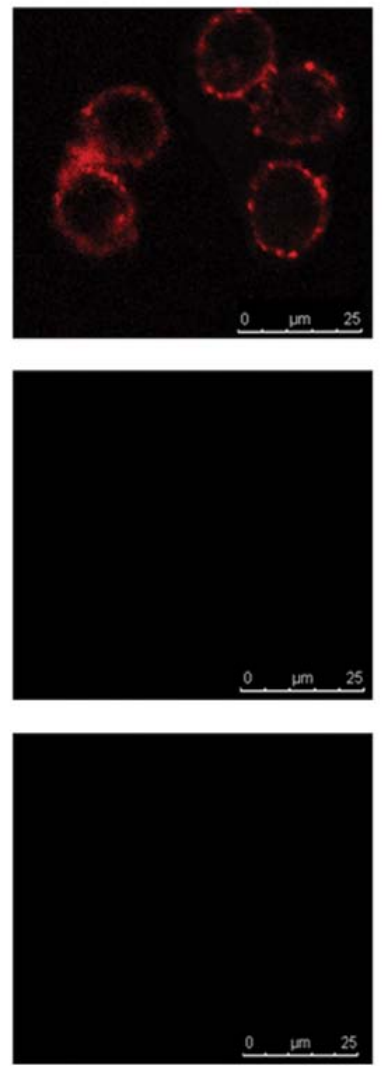

overlay image
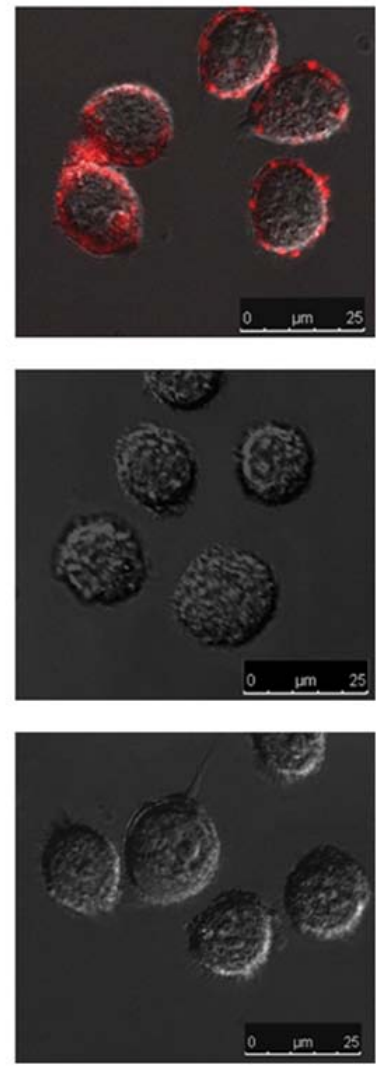

Figure 1. Fluorescent images of U14 cells after 30 min of labeling with QD800-EGFR Ab probes. (A) Experimental group in which the cells were added with QD800-EGFR Ab probes; (B) control group I in which the cells were added with QD800; (C) control group II in which the cells were added with $200 \mu 1$ EGFR monoclonal antibody $(1 \mu \mathrm{g} / \mathrm{ml})$ to block EGFR. After $2 \mathrm{~h}$ of incubation, the cells were washed with PBS 3 times and then added with $100 \mu \mathrm{l}$ QD800-EGFR $\mathrm{Ab}$ probes $(100 \mathrm{nM})$. All images were acquired under the same conditions (excitation/emission, 405/750-800 nm) and displayed on the same scale (scale bar, $25 \mu \mathrm{m})$.

conducted for all the animals after $30 \mathrm{~min}, 1,3,6,9$ and $24 \mathrm{~h}$ of injection of the QD800-EGFR Ab probes or QD800 using Maestro In Vivo imaging system (CRI). Excitation/emission wavelength was $630 / 800 \mathrm{~nm}$, the exposure time was $50 \mathrm{~ms}$ and the acquisition time was $10 \mathrm{sec}$ with a binning of $2 \times 2$ and pixels of $1024 \times 1024$. All the images were processed and the data were analyzed by Maestro2.10.0 software. Auto- and the target-fluorescence were detected and each signal was assigned with a pseudo-color. Auto-fluorescence in this study was set to green color and the target signal was set to red color. Finally, overlay of the two color images were used for analysis.

Cellular and histological examination of the U14 tumors. Two mice from the experimental group, control group I and control groups II, respectively, were euthanized $6 \mathrm{~h}$ after injection of QD800-EGFR Ab probe and QD800. The remaining 3 mice from the experimental and control groups were euthanized $24 \mathrm{~h}$ after of QD800-EGFR Ab probe injection. The subcutaneous neck tumors were excised and embedded in Optimal Cutting Temperature compound. The embedded samples were frozen and cryo-sections with a thickness of $7 \mu \mathrm{m}$ were continuously cut at $-20^{\circ} \mathrm{C}$. One of every two continuous sections was stained with hematoxylin and eosin (H\&E) for observation of the tumor growth. The other section was used for confocal microscopy analysis to observe the distribution of QD800 in the tissues. Confocal parameter settings were the same as described above.

Statistical analysis. Statistical analysis was performed using paired and unpaired Student's t-tests for comparisons between and within groups, respectively. Statistical significance was established at the level of $\mathrm{P}<0.05$. Data are presented as the means $\pm \mathrm{SE}$.

\section{Results}

In vitro labeling of U14 cells by QD800-EGFR Ab probes. Red fluorescence of QD800 was observed on the membrane of U14 cells in the experimental group (Fig. 1A). In contrast, fluorescence was not observed in the cells of control group I or control group II (Fig. 1B and C), suggesting that QD800 does not bind U14 cells, while QD800-EGFR Ab probes can bind U14 cells. In addition, QD800-EGFR Ab probes did not bind U14 cells that had been blocked by EGFR monoclonal antibody. These results demonstrated that the immunological activity of EGFR Ab was maintained after conjugation with QD800 and could specifically recognize EGFR expressed on the surface of U14 cells, leading to the attachment of QD800 to the cell surface. 
A

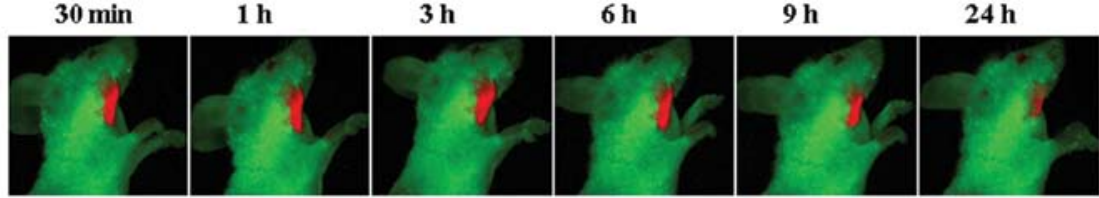

B

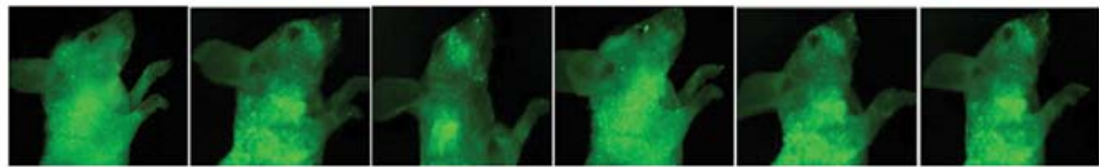

C

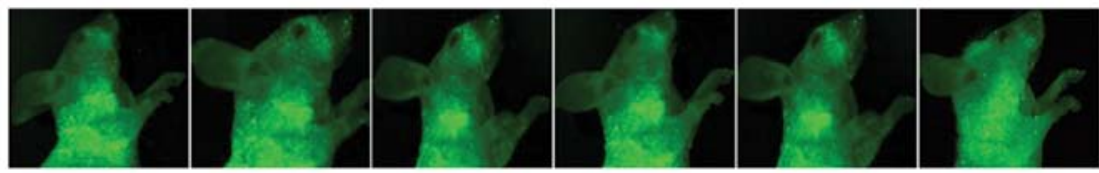

D

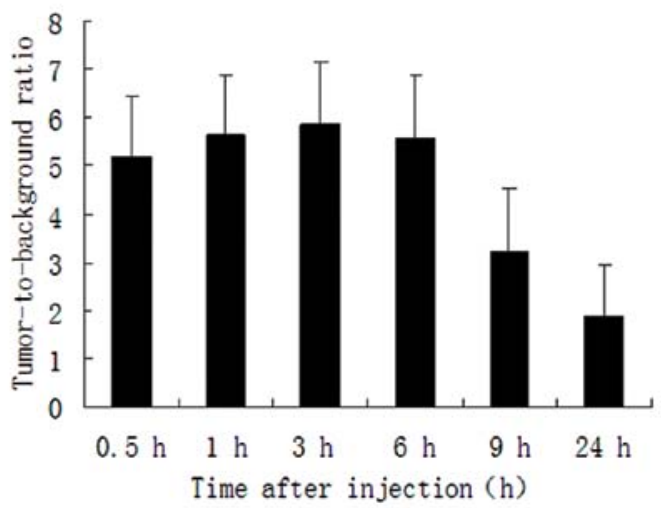

Figure 2. In vivo imaging of tumors by QD800-EGFR Ab probes at different times points. (A) In vivo images of U14 squamous cell carcinoma mice after intravenous injection with QD800-EGFR Ab probe in the experimental group; (B) in vivo images of U14 squamous cell carcinoma mice after intravenous injection with 100 pmol QD800 in the control groupI; (C) in vivo images of U14 squamous cell carcinoma mice in the control group II, the mice were injected with $250 \mu \mathrm{l}$ EGFR monoclonal antibody $(1 \mathrm{mg} / \mathrm{ml})$ and $24 \mathrm{~h}$ later the mice were injected with 1001 QD800-EGFR Ab probes containing 100 pmol equivalent of QD800; (D) the changes of the signal-to-noise ratio at different time points after injection of the probe for in vivo imaging of U14 squamous cell carcinoma in the experimental group.
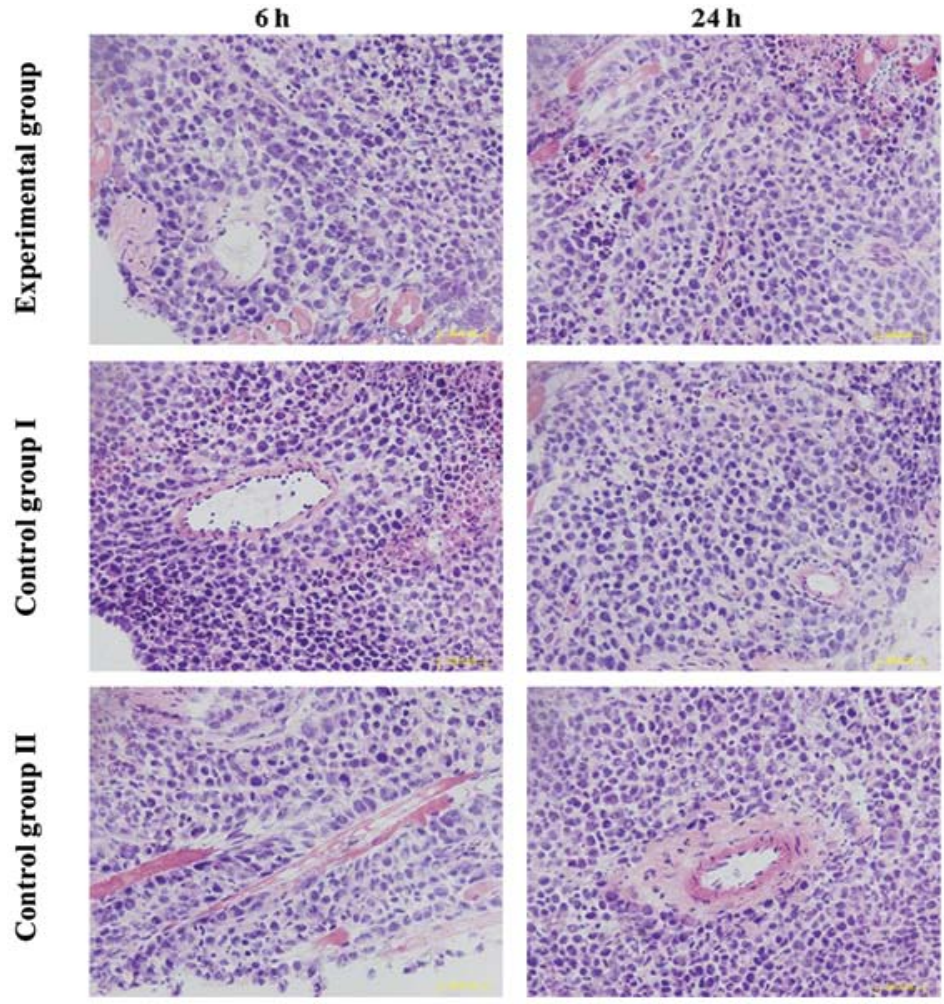

Figure 3. Hematoxylin and eosin staining of tumor sections. H\&E staining of tumor section from the experimental group, control group I and control group II of U14 squamous cell carcinoma after 6 and $24 \mathrm{~h}$ of probe injection. 


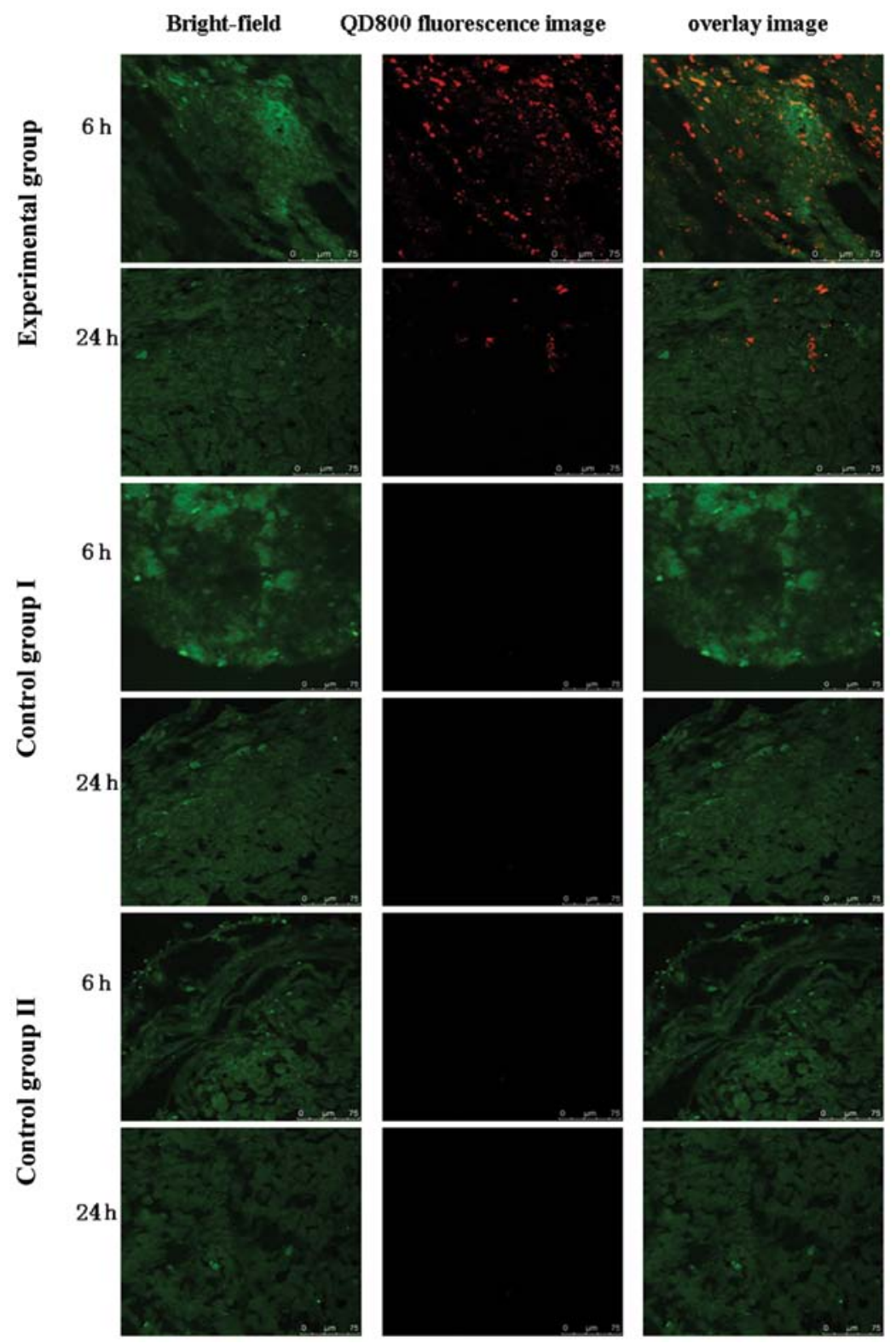

Figure 4. Distribution of fluorescence signals in tumors. Laser Scanning Confocal Microscope analysis of tumor frozen tissue sections from the experimental group, control group I and control group II of U14 squamous cell carcinoma after 6 and $24 \mathrm{~h}$ of probe injection (scale bar, $75 \mu \mathrm{M}$ ).

In vivo imaging of tumors. Significant tumor growth was observed 1 week after inoculation with U14 cells in the chinneck junction area of nude mice. Tumors developed in all the 15 nude mice. Two weeks after inoculation, the maximum diameter of the tumors reached $0.8-1.2 \mathrm{~cm}$ and at this time point we started the imaging experiment. The tumor sites of mice in the experimental group showed significant fluorescent signals 30 min after injection of QD800-EGFR Ab probes via the tail vein. The most complete fluorescent signals were observed after $30 \mathrm{~min}$ to $6 \mathrm{~h}$ of probe injection and the size of the fluorescent images corresponded to that of the tumors. The size of the fluorescent images was significantly reduced after $9 \mathrm{~h}$ of probe injection and the fluorescent images were minimal after $24 \mathrm{~h}$ of probe injection (Fig. 2A). The signal-tonoise ratio (the fluorescence intensity ratio between tumor and background) was relatively high from $30 \mathrm{~min}$ to $6 \mathrm{~h}$ after probe injection. The signal-to-noise ratio was significantly decreased at $9 \mathrm{~h}$ and was close to the baseline level at $24 \mathrm{~h}$ after probe injection (Fig. 2D). In the control group I and control group II, fluorescent signals were not detected at the tumor sites after $30 \mathrm{~min}$ to $24 \mathrm{~h}$ of probe injection (Fig. $2 \mathrm{~B}$ and C).

Cellular and histological examination of the U14 tumors. $\mathrm{H} \& \mathrm{E}$ staining of the tumor sections from the mice after $6 \mathrm{~h}$ and $24 \mathrm{~h}$ of probe injection showed that there were large amount of cancer cells in the tumors of both experimental and control groups, suggesting that tumors were well developed (Fig. 3). Confocal microscopy analysis of the cryo-sections confirmed that QD800 fluorescence signal was not detected in the tumor section of mice in the control group I and control group II after $6 \mathrm{~h}$ and $24 \mathrm{~h}$ of injection. In contrast, in the experimental group, large amount of QD800 was accumulated in the tumor section of mice after $6 \mathrm{~h}$ of injection. QD800 was scattered in the tumor section of mice after $24 \mathrm{~h}$ of injection (Fig. 4). 


\section{Discussion}

Direct in vivo and in situ imaging of cancer cells is one of the key technologies for investigation of tumor development, early diagnosis, drug screening and personalized treatment $(9,10,21,22)$. In comparison to the existing organic fluorescent dyes and fluorescent proteins, QDs have excellent optical properties, e.g., high light stability and high fluorescence quantum yields. Particularly, NIRF-QDs have excellent tissue penetration and great prospects for non-invasive in vivo imaging of the cancer cells (1-4). Previous studies have demonstrated that QDs at the experimental concentration are not cytotoxic and do not affect the growth and differentiation of the live cells $(3,4,23)$. Our previous studies have also shown that labeling of cancer cells with NIRF-QDs do not affect their growth, proliferation, apoptosis, invasion, metastasis or the ability to form tumors (10,24-26). Clinically, optical examination is a safe, simple and economical approach $(18,27)$. In addition to the unique optical properties as described earlier. QDs, as nanoparticles, have an easily modified surface, can be connected to variety of biological molecules and can easily penetrate tumor angiogenesis and reach the cancer cells $(10,16-18)$. Therefore, QDs have shown unique advantages for non-invasive in vivo imaging of cancers.

One of the difficult problems in surgical treatment is how to correctly identify the tumor boundary and determine the scope of tailored surgical resection for different patients to improve their survival rate and quality of life. Since there is no method for direct visualization of cancer cells during surgery, clinicians determine the surgery boundary by experience and the alteration of the cancer tissue texture, which leads to a failure rate of as high as $40 \%$ in patients with complete removal of head and neck cancer surgery $(28,29)$ and greatly affects the patients' survival rate. Therefore developing methods by which clinicians could monitor cancer cells in real time during surgery and perform individual tailored surgical resection is one of the key technologies to improve survival. In this study, we implanted U14 squamous cancer cells with high expression of EGFR into the head and neck sites of nude mice for in vivo imaging studies. This was due: 1), most of the malignant tumors in the head and neck regions are squamous cell carcinoma and $90 \%$ of the squamous cancer cells highly express EGFR $(30,31)$. Therefore, QD in vivo imaging by targeting EGFR has broad applicability for HNSCC; 2), targeting of EGFR antibody-conjugated QDs (a kind of nano-probe) to the tumors after intravenous injection is closely related to the location of the tumors and currently there are no reports with regard to the QDs in vivo imaging of head and neck tumors.

In this study, we conjugated NIRF-QDs with EGFR monoclonal antibody to produce QD800-EGFR Ab probes. Our results demonstrated that QD800-EGFR Ab probes can specifically bind U14 squamous cancer cells both in vivo and in vitro and produce clear images of head-neck tumors. Imaging of U14 squamous cell carcinoma by QD800-EGFR Ab probes is achieved by both active and passive targeting $(18,32)$. The tumor is rich in angiogenesis. The basal membrane of tumor angiogenesis is incomplete or lacking and there are wide gaps between the endothelial cells. The presence of these gaps results in the passive targeting of the QD800-EGFR Ab probes into the tumor tissues through the highly permeable tumor angiogenesis. Specific antigen-antibody binding leads to the active targeting of QD800-EGFR Ab probes to the EGFR on cell surface. Thus, EGFR Ab acts as a bridge to connect QD800 to the cells. Our studies showed that the bright and complete fluorescence images are not significantly changed from $30 \mathrm{~min}$ to $6 \mathrm{~h}$ in the experimental group after intravenous injection of QD800-EGFR Ab probes. However, the size and intensity of the fluorescence images are significantly decreased after $9 \mathrm{~h}$ of probe injection, indicating that the best time for imaging of HNSCC by QD800-EGFR Ab probes is from $30 \mathrm{~min}$ to $6 \mathrm{~h}$ after intravenous injection of the probes. After $24 \mathrm{~h}$ of probe injection, the images of the tumors are further reduced and the intensity of fluorescence is decreased. This might be due to the gradual degradation of ligands and coating layer on the surface of probe by lysosomal enzymes $(18,32,33)$, leading to gradual decreases of the probe binding ability and gradual quenching of the fluorescent quantum dots. Our previous studies (10) have also shown that in vivo imaging can detect a minimum of $10^{4}$ cancer cells labeled with NIRF-QDs in the presence of skin barrier, which was 100 -fold higher than the minimal detection limit of CT and MRI. In addition, the sensitivity can be further enhanced when the tumor is exposed during surgery. Gao et al predicted (32) that in vivo imaging can detect as low as 10-100 QDs-labeled cancer cells. In this study, toxicity of the ODs in the mice was not observed.

This study demonstrates that intravenous injection of QD800-EGFR Ab probes can produce clear in vivo and in situ images of HNSCC with high expression of EGFR. QD800-EGFR Ab probes have shown great potential in the investigation of tumor development, early diagnosis and its individual tailored surgical resection. Previous studies have suggested that biologically functionalized QDs have excellent biological compatible and are water soluble $(3,4,23)$. In addition, they do not cause side effects on live subjects and do not affect cellular growth, differentiation and function within the range of experimental doses $(3,4,10,23-26)$. However, the core of QDs is typically made up of heavy metal elements, e.g., plutonium, cadmium and mercury. These metal elements are toxic when they are released. Though the toxicity of these QDs can be reduced by using core-shell structure, long-term in vivo biological oxidation and degradation may cause $\mathrm{QD}$ shell shedding, which can lead to the release of heavy metal ion and subsequent toxic action (34). Therefore, the studies of replacing plutonium, cadmium or mercury with other elements to produce non-toxic QDs or speeding up excretion of QDs from body are important directions for future investigations.

\section{Acknowledgements}

The study was supported by the National Natural Science Foundation of China (No. 81172205; 30872925).

\section{References}

1. Bentolila LA, Ebenstein Y and Weiss S: Quantum dots for in vivo small-animal imaging. J Nucl Med 50: 493-496, 2009.

2. Ciarlo M, Russo P, Cesario A, Ramella S, Baio G, Neumaier CE and Paleari L: Use of the semiconductor nanotechnologies 'quantum dots' for in vivo cancer imaging. Recent Pat Anticancer Drug Discov 4: 207-215, 2009. 
3. Michalet X, Pinaud FF, Bentolila LA, Tsay JM, Doose S and Li JJ: Quantum dots for live cells, in vivo imaging, and diagnostics. Science 307: 538-544, 2005.

4. Medintz IL, Uyeda HT, Goldman ER and Mattoussi H: Quantum dot bioconjugates for imaging, labelling and sensing. Nat Mater 4: 435-446, 2005.

5. Smith AM, Gao X and Nie S: Quantum dot nanocrystals for in vivo molecular and cellular imaging. Photochem Photobiol 80: 377-385, 2004

6. Gao X, Yang L, Petro JA, Marshall FF, Simons JW and Nie S: In vivo molecular and cellular imaging with quantum dots. Curr Opin Biotechnol 16: 63-72, 2005.

7. Smith AM, Duan H, Mohs AM and Nie S: Bioconjugated quantum dots for in vivo molecular and cellular imaging. Adv Drug Deliv Rev 60: 1226-1240, 2008.

8. Wang $\mathrm{C}$, Gao $\mathrm{X}$ and $\mathrm{Su} \mathrm{X}$ : In vitro and in vivo imaging with quantum dots. Anal Bioanal Chem 4: 1397-1415, 2010

9. Byers RJ and Hitchman ER: Quantum dots brighten biological imaging. Prog Histochem Cytochem 45: 201-237, 2011.

10. Cao Y, Yang K, Li Z, Zhao C, Shi C and Yang J: Near-infrared quantum-dot-based non-invasive in vivo imaging of squamous cell carcinoma U14. Nanotechnology 2: 475104, 2010.

11. Mamot C, Drummond DC, Noble CO, Kallab V, Guo Z, Hong K, Kirpotin DB and Park JW: Epidermal growth factor receptortargeted immunoliposomes significantly enhance the efficacy of multiple anticancer drugs in vivo. Cancer Res 65: 11631-11638, 2005.

12. Koo H, Huh MS, Ryu JH, Lee DE, Sun IC and Choi K, Kim K and Kwon IC: Nanoprobes for biomedical imaging in living systems. Nano Today 6: 204-220, 2011.

13. Kobayashi $\mathrm{H}$ and Choyke PL: Target-cancer-cell-specific activatable fluorescence imaging probes: rational design and in vivo applications. Acc Chem Res 44: 83-90, 2010.

14. Aswathy RG, Yoshida Y, Maekawa T and Kumar DS: Nearinfrared quantum dots for deep tissue imaging. Anal Bioanal Chem 397: 1417-1435, 2010.

15. Jiang W, Singhal A, Kim BYS, Zheng J, Rutka JT and Wang C: Assessing near-infrared quantum dots for deep tissue, organ, and animal imaging applications. J Assoc Lab Autom 13: 6-12, 2008.

16. Shi C, Zhu Y, Xie Z, Qian W, Hsieh CL and Nie S: Visualizing human prostate cancer cells in mouse skeleton using bioconjugated near-infrared fluorescent quantum dots. Urology 74 446-451, 2009

17. Tada $\mathrm{H}$, Higuchi $\mathrm{H}$, Wanatabe $\mathrm{TM}$ and Ohuchi $\mathrm{N}$ : In vivo real-time tracking of single quantum dots conjugated with monoclonal anti-HER 2 antibody in tumors of mice. Cancer Res 67 : 1138-1144, 2007.

18. Diagaradjane P, Orenstein-Cardona JM, Colón-Casasnovas NE, et al: Imaging epidermal growth factor receptor expression in vivo: Pharmacokinetic and biodistribution characterization of a bioconjugated quantum dot nanoprobe. Clin Cancer Res 14: 731-741, 2008

19. DiSilvestro PA, Walker JL, Morrison A, Rose PG, Homesley H and Warshal D: Radiation therapy with concomitant paclitaxel and cisplatin chemotherapy in cervical carcinoma limited to the pelvis: a phase I/II study of the Gynecologic Oncology Group. Gynecol Oncol 103: 1038-1042, 2006.
20. Slichenmyer WJ and Fry DW: Anticancer therapy targeting the erbB family of receptor tyrosine kinases. Semin Oncol 28: 67-79, 2001.

21. Lee S, Park K, Kim K, Choi K and Kwon IC: Activatable imaging probes with amplified fluorescent signals. Chem Commun 36: 4250-4260, 2008

22. Koo H, Lee H,Lee S, Min KH, Kim MS and Lee DS: In vivo tumor diagnosis and photodynamic therapy via tumoral $\mathrm{pH}$-responsive polymeric micelles. Chem Commun 46: 5668-5670, 2010.

23. Jaiswal JK, Mattoussi H, Mauro JM and Simon SM: Long-term multiple color imaging of live cells using quantum dot bioconjugates. Nat Biotechnol 21: 47-51, 2003.

24. Yang K, Li Z, Cao Y, Yu X and Mei J: Effect of peptideconjugated near-infrared fluorescent quantum dots (NIRF-QDs) on the invasion and metastasis of human tongue squamous cell carcinoma cell line Tca8113 in vitro. Int J Mol Sci 10: 4418-4427, 2009.

25. Li ZG, Yang K, Cao YA, Zheng G, Sun DP and Zhao C: In vivo study of the effects of peptide-conjugated near-infrared fluorescent quantum dots on the tumorigenic and lymphatic metastatic capacities of squamous cell carcinoma cell line Tca8113 and U14. Int J Mol Sci 11: 1413-1422, 2010.

26. Sun D, Yang K, Zheng G, Li Z and Cao Y: Study on effect of peptide-conjugated near-infrared fuorescent quantum dots on the clone formation, proliferation, apoptosis, and tumorigenicity ability of human buccal squamous cell carcinoma cell line BcaCD885. Int J Nanomed 5: 401-405, 2010.

27. Chen Y, Intes X and Chance B: Development of high-sensitivity near-infrared fluorescence imaging device for early cancer detection. Biomed Instrum Technol 39: 75-85, 2005.

28. Woolgar JA and Triantafyllou A: A histopathological appraisal of surgical margins in oral and oropharyngeal cancer resection specimens. Oral Oncol 41: 1034-1043, 2005.

29. Rosenthal EL, Kulbersh BD, Duncan RD, Zhang W, Magnuson JS, Carroll WR and Zinn K: In vivo detection of head and neck cancer orthotopic xenografts by immunofluorescence. Laryngoscope 116: 1636-1641, 2006.

30. Rogers SJ, Harrington KJ, Rhys-Evans P, O-Charoenrat P and Eccles SA: Biological significance of c-erbB family oncogenes in head and neck cancer. Cancer Metastasis Rev 24: 47-69, 2005.

31. Kalyankrishna S and Grandis JR: Epidermal growth factor receptor biology in head and neck cance. J Clin Oncol 24: 2666-2672, 2006.

32. Gao X, Cui Y, Levenson RM, Chung LW and Nie S: In vivo cancer targeting and imaging with semiconductor quantum dots. Nat Biotechnol 22: 969-976, 2004.

33. Cai W, Shin DW, Chen K, Gheysens O, Cao Q and Wang SX: Peptide-labeled near-infrared quantum dots for imaging tumor vasculature in living subjects. Nano Lett 6: 6669-6676, 2006.

34. Yong KT: Mn-doped near-infrared quantum dots as multimodal targted probes for pancreatic cancer imaging. Nanotechnology 20: 015102, 2009. 